\title{
Beclin-1 Expression is a Predictor of Clinical Outcome in Patients with Esophageal Squamous Cell Carcinoma and Correlated to Hypoxia-Inducible Factor (HIF)-1 $\alpha$ Expression
}

\author{
Yongshun Chen $\cdot$ You Lu $\cdot$ Changli Lu $\cdot$ Lei Zhang
}

Received: 1 September 2008 / Accepted: 16 December 2008 / Published online: 9 January 2009

(C) The Author(s) 2009. This article is published with open access at Springerlink.com

\begin{abstract}
In the present study, we examined the relationship between Beclin-1 expression and HIF- $1 \alpha$ expression in esophageal squamous cell carcinoma(ESCC). There was a loss of Beclin-1 protein expression in 33\% of ESCCs. Beclin-1 expression significantly correlated with depth of invasion, lymph node metastasis and clinical stage. Among the 54 patients, The survival rate of the Beclin-1-positive group was better than that of the Beclin-1-negative group. Twenty-five of the 54 (46\%) tumor specimens showed high levels of HIF-1 $\alpha$ immunoreactivity. Beclin-1 expression was associated with HIF- $1 \alpha$ expression. The survival rate of patients with Beclin-1-positive and HIF- $1 \alpha$-low tumors was significantly higher than that of the other groups. These
\end{abstract}

Y. Chen · Y. Lu $(\bowtie)$

Division of Thoracic Oncology, West China Hospital, Cancer Center, West China School of Clinical Medicine,

Sichuan University,

37, Guoxue Lane,

Chengdu 610041, China

e-mail: radyoulu@hotmail.com

Y. Chen

e-mail: yongshun2007@gmail.com

Y. Chen · Y. Lu

State Key Laboratory of Biotherapy, West China Hospital, Sichuan University,

Chengdu, China

C. $\mathrm{Lu}$

Department of Pathology, West China Hospital,

West China School of Clinical Medicine, Sichuan University,

Chengdu, China

\section{Zhang}

Department of Nephrology, West China Hospital,

West China School of Clinical Medicine, Sichuan University,

Chengdu, China results suggest that Beclin-1 and HIF- $1 \alpha$ expression are important determinants of survival in ESCCs.

Keywords Esophageal squamous cell carcinoma .

Beclin- 1 HIF-1 $\alpha \cdot$ Immunohistochemistry ·

Clinicopathological factors $\cdot$ Survival

\section{Introduction}

Programmed cell death (PCD) is an essential and highly orchestrated process that plays a major role in morphogenesis and tissue homeostasis. Apoptosis, the type I PCD, is a well characterized caspase-dependent process. While autophagy, a vacuolar process of cytoplasmic degradation, is characterized by autophagic vacuoles accumulating, no apoptosis body forming, and no chromosome condensing and caspase independence. Thus, autophagic cell death is considered PCD type II $[1,2]$. Autophagy is becoming an important area in cancer research, but data connecting cancer pathogenesis with autophagic cell death are limited. Whether autophagy in cancer cells causes death or protects cells is controversial. Malignant transformation is frequently associated with suppression of autophagy, however, the opposite scenario has also been reported [3, 4].

Beclin-1, an essential mediator of autophagy, was originally discovered during the course of a yeast two-hybrid screen of a mouse brain cDNA library [5]. The human Beclin1 gene has been mapped to a tumor-susceptibility locus on chromosome $17 \mathrm{q} 21$ that is monoallelically deleted in up to $75 \%$ of ovarian cancers, $50 \%$ of breast cancers, and $40 \%$ of prostate cancers [6]. Moreover, Beclin- $1^{-/-}$mutant mice die early in embryogenesis and Beclin- $1^{+/-}$mutant mice suffer from a high incidence of spontaneous tumors, establishing that Beclin-1 is a critical component of mammalian autophagy 
and a role for autophagy in tumor suppression [7]. Liang's group [8] found a frequent loss of Beclin-1 protein expression in breast cancers, and suggested that tumor suppressor functions of Beclin-1 are lost in the cancer cells by loss of protein expression. Ahn et al. [9] demonstrated that Beclin-1 may play a role in tumorigenesis for the increased expression of Beclin-1 protein detected in the malignant colorectal and gastric epithelial cells. These contradictory results prompt us to analyse Beclin-1 protein expression in human esophageal cancer tissues.

HIF- $1 \alpha$ has been found to play important roles in tumor growth and metastasis by regulating energy metabolism and inducing angiogenesis in the survival against cellular hypoxia. In various types of cancer, HIF- $1 \alpha$ expression was reported to be correlated with tumor growth, invasion and metastases [10-13]. It was reported that autophagy associates with hypoxia. Autophagic cell death is induced by hypoxia in cancer cells with intact apoptotic machinery, and HIF-1 serves to regulate autophagy in mouse embryo fibroblasts [14-16]. However, there are no reports with respect to the relationship between Beclin-1 and HIF- $1 \alpha$ expressions in human cancers. We therefore examined their expressions in human squamous cell carcinoma of the esophagus. The primary objective was to ascertain whether Beclin-1 plays a role in tumor development and progression associated with HIF- $1 \alpha$, as secondary endpoints, we evaluated the impact of their protein expressions on patient outcome.

\section{Materials and Methods}

\section{Patients and Tissues}

54 cases of esophagectomy specimens of primary esophageal squamous cell carcinoma (ESCC) were collected from the files of the Department of Pathology, West China Hospital, Sichuan University, dating from January 2003 to September 2004. All patients did not receive preoperative chemoradiotherapy. This study was conducted in accordance with ethical principles stated in the Declaration of Helsinki, 1996 and informed consent for using esophageal tissues was obtained from the patients or their families.

To determine an accurate staging, pathology reports and clinical histories at the time of surgery were reviewed. The clinicopathologic stage was according to the International Union Against Cancer (UICC) TNM classification system [17]. Age, sex, tumor differentiation and TNM stage (T: depth of tumor invasion, $\mathrm{N}$ : lymph node metastasis, M: distant metastasis) were evaluated. The overall survival times of the patients were measured from the date of operation to the date of death or the last follow-up. The patients were 38 males and 16 females and ranged in age from 39 to 77 years (median: 61 years).
Immunohistochemistry

Formalin-fixed, paraffin-embedded tissues ( $4 \mu \mathrm{m}$ thick) were immunostained using a Labelled Streptavidin Biotin 2 (LSAB2) System (BioGenex i6000, USA). Beclin-1 was detected with a rabbit polyclonal antibody and HIF-1 $\alpha$ was detected with a mouse monoclonal antibody Mab (1:80 dilution for the anti-Beclin-1 antibody or 1:100 dilution for the anti-HIF-1 $\alpha$ antibody; Santa Cruz Biotechnology, Santa Cruz, USA). After deparaffinization and rehydration, tissue sections were incubated with $3 \%$ hydrogen peroxide in methanol for $30 \mathrm{~min}$ to block endogeneous peroxidase activity. The primary antibody reaction was carried out at $4{ }^{\circ} \mathrm{C}$ overnight. Subsequent steps were performed according to the manufacturer's instructions. Diaminobenzidinetetrahydrochloride (DAB) was used as the substrate to detect antigen-antibody binding, and the sections were counterstained with Mayer's haematoxylin.

Beclin-1 and HIF- $1 \alpha$ immunoreactivity were detected in normal epithelial cells of esophagus and squamous cell carcinoma tissues. Expression of Beclin-1 was defined as positive if distinct staining of the cytoplasm or cytomembrane was observed in at least $10 \%$ of tumor cells.

HIF- $1 \alpha$ immunoreactivity was present in cytoplasm. The percentages of cancer cells with cytoplasmic HIF- $1 \alpha$ localisation were assessed separately in all optical fields. The mean value of the percentages obtained from all optical fields examined in each case was used to calculate the cytoplasmic scores for each case. These scores were used to calculate the overall mean value and range of the percentages of cells with HIF- $1 \alpha$ localisation. The mean values for cytoplasmic expression of HIF- $1 \alpha$ were used as the cut-off points to define two groups: high and low HIF-1 $\alpha$ expression.

\section{Statistical Analysis}

Statistical evaluation was performed using the SPSS software package (SPSS 16.0, SPSS Inc., Chicago, USA). The Chi-square test was used to analyze the correlation between Beclin-1 or HIF- $1 \alpha$ expression and clinicopathologic features. Cumulative survival rate was calculated by the Kaplan-Meier method, and the significance of differences in survival rate was analyzed by the log-rank test. The level of statistical significance was set at $p<0.05$.

\section{Results}

Relationship between Beclin-1 Expression and Clinicopathological Features

Of the 54 ESCCs, immunoreactivity for Beclin-1 was not detected in 18 cases (Fig. 1b). 36 (66.7\%) showed positive 

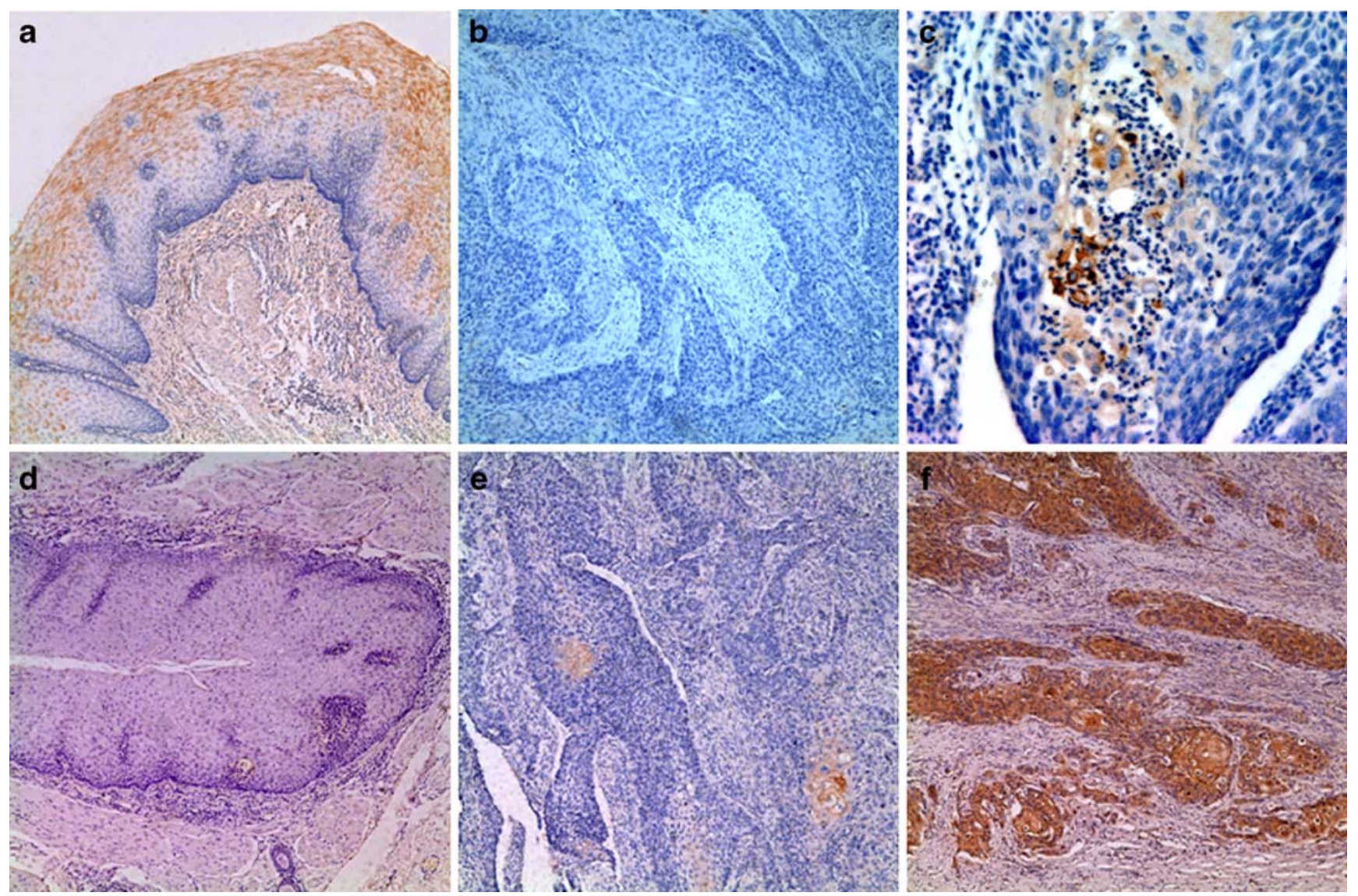

Fig. 1 Immunohistochemical analysis of Beclin-1 and HIF- $1 \alpha$ in esophageal squamous cell carcinoma. a Positive Beclin-1 immunoreactivity in cytoplasm of normal squamous epithelial cells. b Negative Beclin-1 immunoreactivity in tumour cells. c Positive Beclin-1 immunoreactivity in cytoplasm and cytomembrane of tumour cells.

Beclin-1 immunoreactivity in the cytoplasm and cytomembrane of cancer cells (Fig. 1c). While analyzing clinicopathological factors, depth of invasion, lymph node metastasis and clinical stage showed statistically significant correlations with Beclin-1 expression; however, there was no significant difference in Beclin-1 expression with respect to other characteristics (sex, age and differentiation) (Table 1). Beclin-1 was displayed positive immunoreactivity in normal epithelial cells of all cases adjacent to squamous cell carcinoma tissues (Fig. 1a).

\section{Relationship between HIF-1 $\alpha$ Expression} and Clinicopathological Features

HIF- $1 \alpha$ immunoreactivity was not detected in normal squamous cells of esophagus (Fig. 1d). HIF-1 $\alpha$ staining was observed mainly in the cytoplasm of the esophageal cancer cells (Fig. 1e, f). The mean percentage of cells with cytoplasmic HIF- $1 \alpha$ immunoreactivity was $28.2 \%$ (range $5-80 \%)$. On the basis of the criteria described in Section 2, d In normal squamous epithelial cells, HIF- $1 \alpha$ immunoreactivity was not detected. e Weak HIF- $1 \alpha$ immunoreactivity in cytoplasm of tumour cells. f Strong HIF-1 $\alpha$ immunoreactivity in tumour cells. Original magnification: a, e and f, $\times 200 ; \mathbf{b}$ and $\mathbf{d}, \times 100 ; \mathbf{c}, \times 400$

25 of the $54 \operatorname{ESCCs}(46.3 \%)$ were judged to have high HIF- $1 \alpha$ expression. HIF- $1 \alpha$ immunoreactivity was correlated significantly with differentiation, depth of invasion, and clinical stage (Table 2).

\section{Relationship between Beclin-1 and HIF-1 $\alpha$ Expression}

We analysed the association between Beclin- 1 and HIF- $1 \alpha$ protein expression. In the 36 ESCCs marked as Beclin1-positive tumors, 24 were low-HIF- $1 \alpha$ and 12 were high-HIF- $1 \alpha$. Whereas, in the 18 ESCCs classified as Beclin-1-negative tumors, the low-HIF- $1 \alpha$ and high-HIF$1 \alpha$ tumors were 5 and 13 , respectively. A significant association was found between Beclin-1 and HIF- $1 \alpha$ expression $(P=0.001$, Table 3$)$.

\section{Cumulative Kaplan-Meier Survival Curve}

The median follow-up period was 33.5 months with a range from 11 to 56 months. The 1-, 2- and 3-year survival for 
Table 1 Clinicopathological features and Beclin-1 expression in ESCC

\begin{tabular}{lcccc}
\hline Variables & No. of cases & \multicolumn{2}{l}{ Beclin-1 expression } & \multirow{2}{*}{$P$ value } \\
\cline { 3 - 3 } & & Positive & Negative & \\
\hline Gender & & & & \\
Male & 38 & 24 & 14 & 0.399 \\
Female & 16 & 12 & 4 & \\
Age (years) & & & & \\
$<60$ & 30 & 20 & 10 & 1.00 \\
$\geq 60$ & 24 & 16 & 8 & \\
Differentiation & & & & \\
Well & 12 & 10 & 2 & 0.351 \\
Moderate & 20 & 13 & 7 & \\
Poor & 22 & 13 & 9 & \\
T stage & & & & \\
T1, T2 & 25 & 23 & 2 & 0.001 \\
T3, T4 & 29 & 13 & 16 & \\
N stage & & & & \\
N0 & 26 & 24 & 2 & $<0.001$ \\
N1 & 28 & 12 & 16 & \\
Clinical stage & & & & \\
I, II & 22 & 21 & 1 & $<0.001$ \\
III, IV & 32 & 15 & 17 & \\
\hline
\end{tabular}

the entire patient group was $95.8 \%, 82.9 \%$ and $56.1 \%$, respectively. The survival rate of the Beclin-1-positive group was better than that of the Beclin-1-negative group (Fig. 2a). Furthermore, there was a tendency toward a

Table 2 Clinicopathological features and HIF-1 $\alpha$ expression in ESCC

\begin{tabular}{lcrrr}
\hline Variables & \multirow{2}{*}{ No. of cases } & \multicolumn{2}{c}{ HIF-1 $\alpha$ expression } & \multirow{2}{*}{$P$ value } \\
\cline { 3 - 4 } & & Low & High & \\
\hline Gender & & & & \\
Male & 38 & 23 & 15 & 0.121 \\
Female & 16 & 6 & 10 & \\
Age (years) & & & & \\
$<60$ & 30 & 13 & 17 & 0.088 \\
$\geq 60$ & 24 & 16 & 8 & \\
Differentiation & & & & \\
Well & 12 & 10 & 2 & 0.003 \\
Moderate & 20 & 13 & 7 & \\
Poor & 22 & 6 & 16 & \\
T stage & & & & \\
T1, T2 & 25 & 24 & 1 & $<0.001$ \\
T3, T4 & 29 & 5 & 24 & \\
N stage & & & & \\
N0 & 26 & 17 & 9 & 0.097 \\
N1 & 28 & 12 & 16 & \\
Clinical stage & & & & \\
I, II & 22 & 17 & 5 & 0.004 \\
III, IV & 32 & 12 & 20 & \\
\hline
\end{tabular}

Table 3 Coincidental expressions of Beclin- 1 and HIF- $1 \alpha$ proteins in ESCC

\begin{tabular}{llll}
\hline & \multicolumn{2}{l}{ Beclin-1 } & \multirow{2}{*}{$P$ value } \\
\cline { 2 - 3 } & Positive & Negative & \\
\hline Low HIF-1 $\alpha$ & $24(36.9 \%)$ & $5(7.69 \%)$ & 0.001 \\
High HIF-1 $\alpha$ & $12(18.5 \%)$ & $13(20 \%)$ & \\
\hline
\end{tabular}

poorer outcome in patients with HIF- $1 \alpha$-high tumors compared with that in patients with HIF- $1 \alpha$-low tumors, however, differences were not statistically significant (Fig. 2b). Bivariate analysis was also assessed in 37 patients. The survival rate of patients with Beclin-1-positive and HIF- $1 \alpha$-low tumors $(n=24)$ was significantly higher than that of the other group $(n=13)$ (Fig. 2c).

\section{Discussion}

Study showed that malignant cells frequently displayed lower levels of basal autophagic activity than their normal counterparts and failed to increase autophagic activity; in addition, Beclin-1, an autophagy execution protein, was viewed as a tumor suppressor protein [18]. In 1999, Liang et al. [8] found that in $56 \%$ of breast cancers there was a significant loss of Beclin-1 protein expression in cancer cells compared to normal breast epithelial cells, later studies reported that Beclin-1 was monoallelically deleted in human prostate, cervical and ovarian cancers and was expressed at reduced levels in those tumors [6, 8, 19]. Miracco et al. [20] recently examined the expression of Beclin-1 protein in 212 primary human brain tumors, in most high-grade astrocytic, ependymal neoplasms and atypical meningiomas they found a decrease of Beclin-1 protein expression that was, instead, high in the majority of low-grade tumors and in medulloblastomas. Which indicates that Beclin-1 expression is related to the aggressiveness of brain tumors.

Furthermore, controversy regarding the roles of autophagy in cancer has been emerged, studies showed that Beclin-1 play different roles in cell death and cell survival depending on the cellular context [21-23]. Koneri and his colleagues [24] investigated the function of Beclin-1 gene in colorectal cancer cell lines, transfection of the low Beclin-1 geneexpressing colon cancer cell line with the Beclin-1 gene resulted in cell growth inhibition, they concluded that Beclin-1 can inhibit the growth of colorectal cancer cells. Wang et al. [18] detected the role of Beclin-1 in HeLa cells using RNA interference and they found that overexpression of Beclin-1 promoted the autophagy cell death but siRNA against Beclin-1 transfectants promoted 

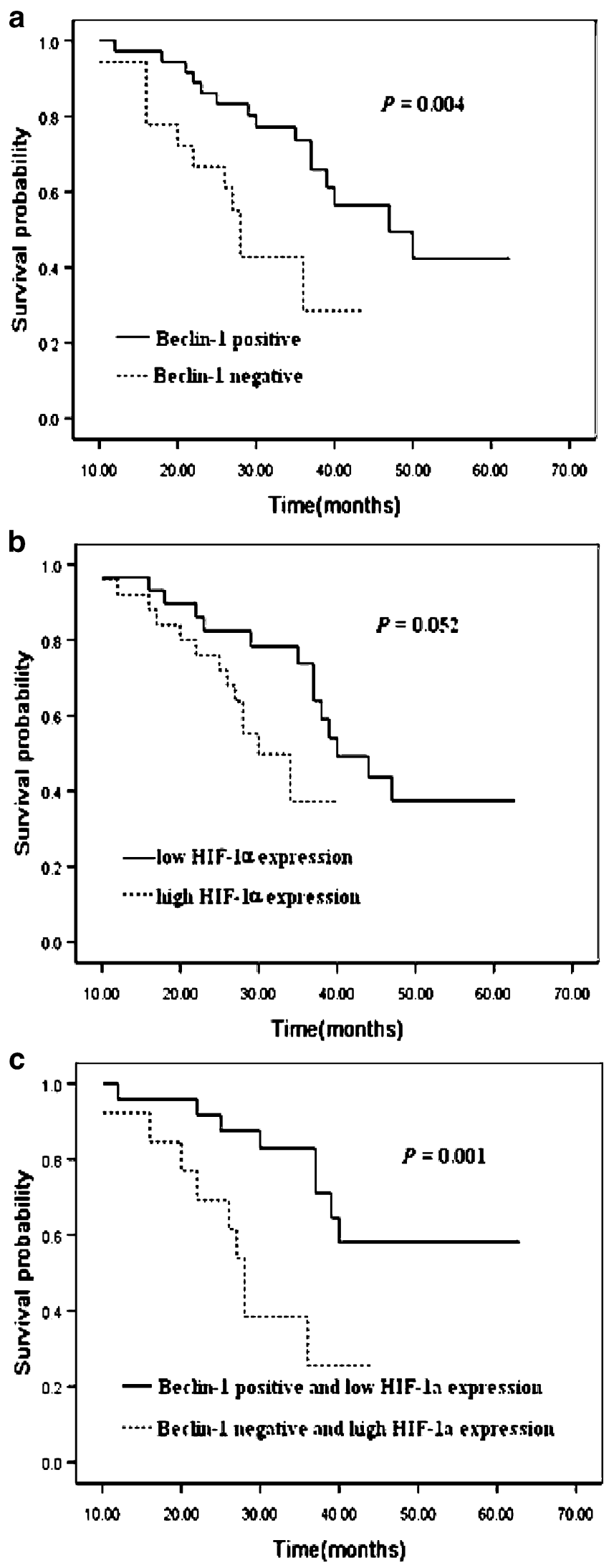

Fig. 2 Cumulative Kaplan-Meier survival curves. a Curves for patients with Beclin-1-positive tumors and Beclin-1-negative tumors. b Curves for patients with HIF- $1 \alpha$-low tumors and HIF- $1 \alpha$-high tumors. c Curves for patients with Beclin-1-positive and HIF- $1 \alpha$-low tumors compared to patients with Beclin-1-negative and HIF-1 $\alpha$ high tumors

the cell proliferation. These examples favor a cancerkilling role for autophagy, overexpression of Beclin-1 induces cell death and defection of autophagy may be an important mechanism in tumorigenesis. However, Amaravadi [25] presented results supporting the idea that autophagy can protect tumor cells from cell death stimuli. Daniel [23] discovered that blockade of Beclin-1 expression aggravates cell death in HepG2 cells. Ahn et al. [9] investigated Beclin1 protein expression in colorectal and gastric carcinoma tissues, there was increased expression of Beclin-1 in the malignant colorectal and gastric epithelial cells compared to their normal mucosal epithelial cells. These studies provide evidence that autophagy serves as a survival pathway in tumor cells.

We detected Beclin-1 protein in squmous cell carcinoma tissues of esophagus for the first time and found that there was a loss of Beclin-1 protein expression in 33\% of ESCCs compared to normal esophageal epithelial cells. Moreover, Beclin-1 immunoreactivity was correlated significantly with depth of invasion, lymph node metastasis and clinical stage, the survival curve of the Beclin-1 negative group was worse than that of the Beclin-1 positive group. These findings may indicate that Beclin-1 plays an important role in development and progression of ESCC and is a factor significantly influencing overall survival rate.

Studies revealed that HIF- $1 \alpha$ expression was absent in most normal tissues, and in human tumors, overexpression of HIF- $1 \alpha$ activated metabolic and pathogenic pathways that were related to tumor angiogenesis, growth, invasion and metastasis [10, 26, 27]. Moreover, HIF-1 $\alpha$ expression may play an important role in the progression of ESCC and is associated with poor patient prognosis $[11,12]$. In the present study, HIF-1 $\alpha$ immunoreactivity was not detected in normal squamous esophageal epithelial cells and HIF- $1 \alpha$ expression was correlated significantly with differentiation, depth of invasion, and clinical stage of ESCC. Our findings are consistent with results from these previous studies. There was a tendency for shorter survival in patients with high HIF- $1 \alpha$ expression, but this correlation did not reach statistical significance, probably owing to short periods of follow-up and the small number of patients enrolled in our study.

Certain environmental cues (such as starvation, low oxygen, hormonal stimulation) activate signalling pathways that increase autophagy, which can either remove defective structures or be as a means of providing macromolecules 
for energy generation. HIF-1 activates transcription and plays a critical role in oxygen homeostasis [28]. The relationship between autophagy and hypoxia has been interpreted. Previous study showed that autophagy is associated with hypoxia during cardiac ischemia and metabolic stress as a survival mechanism [29]. Azad et al. [14] recently analyzed glioma cell lines, breast cancer cell lines and embryonic cell line for cell death response in hypoxia. Under hypoxic conditions, cell lines induced an autophagic response and underwent cell death. By knocking down the autophagy protein Beclin-1, hypoxia-induced cell death was reduced, hypoxia-induced cell death was also reduced upon treatment with the autophagy inhibitor 3methyladenine. This demonstrated that autophagic cell death is induced by hypoxia in cancer cells. Zhang and his colleagues [15] found that mitochondrial autophagy is an adaptive metabolic response which is necessary to maintain redox homeostasis and cell survival. In mouse embryo fibroblasts, HIF-1 induces expression of BNIP3, which triggers selective mitochondrial autophagy. Hypoxia can induce mitochondrial autophagy and this process requires the HIF-1-dependent expression of BNIP3 and the constitutive expression of Beclin-1. Which suggests a close relationship exists between Beclin-1 and HIF-1 [30].

There have been no reports on the association between Beclin-1 and HIF- $1 \alpha$ expression. Interestingly, Beclin-1 expression was associated with HIF- $1 \alpha$ expression in our immunohistochemical analysis. HIF- $1 \alpha$ expression was high while there was reduced expression of Beclin-1 in late stage of ESCC, the survival curve of the Beclin-1 negative and high-HIF-1 $\alpha$ group was worse than that of the Beclin-1 positive and low-HIF-1 $\alpha$ group. This study indicates that Beclin-1 and HIF- $1 \alpha$ proteins may play important roles in the progression of ESCC and are factors significantly influencing overall survival rate. There was a tendency of Beclin-1-induced autophagy inhibition by high expression of HIF-1 $\alpha$, which promotes tumor progression. Additional studies are needed to clarify these association.

In conclusion, we found that Beclin-1 expression is associated with HIF- $1 \alpha$ expression. The Beclin- 1 and HIF$1 \alpha$ proteins appear to play important roles in the progression of human ESCC. Measuring Beclin- 1 and HIF- $1 \alpha$ expression may contribute to a better understanding of the prognosis of patients with ESCC, at least in a subset of patients who had not received preoperative chemoradiotherapy. Beclin-1 can be a potential target for the treatment of ESCCs.

Open Access This article is distributed under the terms of the Creative Commons Attribution Noncommercial License which permits any noncommercial use, distribution, and reproduction in any medium, provided the original author(s) and source are credited.

\section{References}

1. Guimaraes CA, Linden R (2004) Programmed cell death. Apoptosis and alternative deathstyles. Eur J Biochem 271:1638-1650

2. Edinger AL, Thompson CB (2004) Death by design: apoptosis, necrosis and autophagy. Curr Opin Cell Biol 16:663-669

3. Baehrecke EH (2005) Autophagy: dual roles in life and death? Nat Rev Mol Cell Biol 6:505-510

4. Levine B (2007) Autophagy and cancer. Nat Cell Biol 446:745747

5. Liang XH, Kleeman LK, Jiang HH et al (1998) Protection against fatal Sindbis virus encephalitis by beclin 1, a novel Bcl-2 interacting protein. J Virol 72:8586-8596

6. Aita VM, Liang XH, Murty VV et al (1999) Cloning and genomic organization of beclin 1, a candidate tumor suppressor gene on chromosome 17q21. Genomics 59:59-65

7. Yue Z, Jin S, Yang C et al (2003) Beclin 1, an autophagy gene essential for early embryonic development, is a haploinsufficient tumor suppressor. Proc Natl Acad Sci USA 100:15077-15082

8. Liang XH, Jackson S, Seaman M et al (1999) Induction of autophagy and inhibition of tumorigenesis by beclin 1 . Nature 402:672-676

9. Ahn CH, Jeong EG, Lee JW et al (2007) Expression of beclin-1, an autophagy-related protein, in gastric and colorectal cancers. APMIS 115:1344-1349

10. Koukourakis MI, Giatromanolaki A, Sivridis E et al (2002) Hypoxia-inducible factor (HIF $1 \alpha$ and HIF2 $\alpha$ ), angiogenesis and chemoradiotherapy outcome of squamous cell head-and-neck cancer. Int J Radiat Oncol Biol Phys 53:1192-1202

11. Katsuta M, Miyashita M, Makino H et al (2005) Correlation of hypoxia inducible factor- $1 \alpha$ with lymphatic metastasis via vascular endothelial growth factor-C in human esophageal cancer. Exp Mol Pathol 78:123-130

12. Kimura S, Kitadai Y, Tanaka S et al (2004) Expression of hypoxia-inducible factor (HIF)-1 alpha is associated with vascular endothelial growth factor expression and tumour angiogenesis in human oesophageal squamous cell carcinoma. Eur J Cancer 40:1904-1912

13. Enatsu S, Iwasaki A, Shirakusa $T$ et al (2006) Expression of hypoxia-inducible factor-1 alpha and its prognostic significance in small-sized adenocarcinomas of the lung. Eur J Cardiothorac Surg 29:891-895

14. Azad MB, Chen Y, Henson ES et al (2008) Hypoxia induces autophagic cell death in apoptosis-competent cells through a mechanism involving BNIP3. Autophagy 4:195-204

15. Zhang H, Bosch-Marce M, Shimoda LA et al (2008) Mitochondrial autophagy is an HIF-1-dependent adaptive metabolic response to hypoxia. J Biol Chem 283:10892-10903

16. Semenza GL (2008) Mitochondrial autophagy: life and breath of the cell. Autophagy 4:534-536

17. Sobin LH, Fleming ID (1997) TNM classification of malignant tumors, fifth Edition, Union Internationale Contre le Cancer and the American Joint Committee on Cancer. Cancer 80:18031804

18. Gozuacik D, Kimchi A (2004) Autophagy as a cell death and tumor suppression mechanism. Oncogene 23:2891-2906

19. Wang $\mathrm{ZH}, \mathrm{Xu} \mathrm{L}$, Duan $\mathrm{ZL}$ et al (2007) Beclin 1-mediated macroautophagy involves regulation of caspase- 9 expression in cervical cancer HeLa cells. Gynecol Oncol 107:107-113

20. Miracco C, Cosci E, Oliveri G et al (2007) Protein and mRNA expression of autophagy gene Beclin 1 in human brain tumours. Int J Oncol 30:429-436

21. Furuya D, Tsuji N, Yagihashi A et al (2005) Beclin 1 augmented cis-diamminedichloroplatinum induced apoptosis via enhancing caspase-9 activity. Exp Cell Res 307:26-40 
22. Marx J (2006) Autophagy: is it cancer's friend or foe? Science 312:1160-1161

23. Daniel F, Legrand A, Pessayre D et al (2006) Partial Beclin 1 silencing aggravates doxorubicin- and Fas-induced apoptosis in HepG2 cells. World J Gastroenterol 12:2895-2900

24. Koneri K, Goi T, Hirono Y et al (2007) Beclin 1 gene inhibits tumor growth in colon cancer cell lines. Anticancer-Res 27:1453-1457

25. Amaravadi RK, Yu D, Lum JJ et al (2007) Autophagy inhibition enhances therapy-induced apoptosis in a Myc-induced model of lymphoma. J Clin Invest 117:326-336

26. Zhong H, De Marzo AM, Laughner E et al (1999) Overexpression of hypoxia-inducible factor $1 \alpha$ in common human cancers and their metastases. Cancer Res 59:5830-5835
27. Birner P, Schindl M, Obermair A et al (2000) Overexpression of hypoxia-inducible factor $1 \alpha$ is a marker for an unfavorable prognosis in early-stage invasive cervical cancer. Cancer Res 60:4693-4696

28. Iyer NV, Kotch LE, Agani F et al (1998) Cellular and developmental control of $\mathrm{O}_{2}$ homeostasis by hypoxia-inducible factor $1 \alpha$. Genes Dev 12:149-162

29. Brahimi-Horn MC, Pouysségur J (2007) Harnessing the hypoxiainducible factor in cancer and ischemic disease. Biochem Pharmacol 73:450-457

30. Bohensky J, Shapiro IM, Leshinsky S et al (2007) HIF regulation of chondrocyte apoptosis: induction of the autophagic pathway. Autophagy 3:207-214 